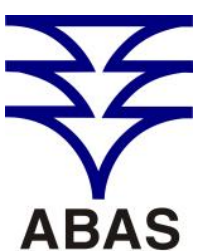

ASSOCIACAOBRASLERRADE
AGUAS SUBTERRANEAS www.abas.org

\title{
PERMEABILIDADE RELATIVA EM ZONA VADOSA COM POROSIDADE BIMODAL: UM ESTUDO EM SOLOS BRASILEIROS
}

\author{
RELATIVE PERMEABILITY IN VADOSE ZONE WITH BIMODAL SOIL \\ POROSITY: A BRAZILIAN SOIL STUDY
}

\author{
Miguel Alfaro Soto'1, Chang Hung Kiang²
}

Artigo recebido em: 03/09/2012 e aceito para publicação em: 09/08/2013.

\begin{abstract}
The present study evaluate the characteristics of the transport of wetting fluid in a vadose zone with bimodal pore-size distributions, characteristic of certain structured soils and rocks. When such heterogeneity is ignored in the traditional theoretical estimation of relative permeability of water, inaccurate predictions will result. Data about bimodal porosity from typical soils of the states of São Paulo and Paraná were used here to estimate relative permeability, using both alternative and traditional methods. The comparative analysis showed that the use a relative permeability model for a soil with bimodal pore-size distributions may result in errors of more than two orders of magnitude; these results highlights the importance of using relative permeability models that take into account the bimodal pore-size distribution.
\end{abstract}

Keywords: Relative permeability. Bimodal soil porosity. Water flow in vadose zone.

Resumo: Neste trabalho são avaliadas as características de transporte de fluidos molhantes em zona vadosa com distribuição de tamanhos dos poros bimodal, comumente encontrados em rochas ou solos estruturados. Essa heterogeneidade não é em geral considerada na estimativa da permeabilidade relativa da água, podendo induzir a erros consideráveis. Utilizando dados de alguns solos típicos do estado de São Paulo e Paraná com porosidade bimodal, são aqui apresentadas estimativas da permeabilidade relativa por métodos alternativos, e também por métodos tradicionais. A análise comparativa mostrou que a utilização de um modelo de permeabilidade relativa tradicional em solos com distribuição de poros bimodal poderá incorrer em erros de mais de duas ordens de magnitude, evidenciando a importância da utilização de modelos de permeabilidade relativa que levem em consideração a bimodalidade da distribuição de tamanho dos poros.

Palavras-chave: Permeabilidade relativa. Porosidade bimodal em solos. Transporte de água em zona vadosa.

\section{INTRODUÇÃO}

Ao longo dos últimos anos, pesquisas relacionadas às propriedades mecânicas e hidráulicas da zona vadosa têm se intensificado. O interesse nesse tipo de estudo reside na crescente necessidade de desenvolver ferramentas para avaliar problemas ambientais, tais como contaminação de solos e águas subterrâneas, geralmente decorrentes de diversos tipos de derramamentos acidentais.

Modelos matemáticos e resultados de trabalhos experimentais constituem, em conjunto, ferramentas de grande ajuda e geralmente se valem dos mais importantes fatores que governam o movimento de água no solo não saturado, tais como as curvas de retenção de água no solo $(\mathrm{h}(\theta))$ e a da condutividade hidráulica não saturada $(\mathrm{k}(\theta))$.

Embora exista um grande número de mé- todos de determinação em campo e laboratório para os parâmetros de $\mathrm{h}(\theta)$ e $\mathrm{k}(\theta)$, a utilização de métodos indiretos tem se intensificado. Atualmente, este tipo de expediente é muito difundido para determinação da função condutividade hidráulica em zona não saturada, que é obtida a partir de modelos que descrevem a curva de retenção da água do solo. Segundo Mallants et al. (1997), devido à sua praticidade, os métodos indiretos economizam tempo. No entanto, essas estimativas podem diferir significativamente dos dados experimentais quando a descrição funcional da curva de retenção não é adequada.

Causas para resultados indesejáveis podem ser atribuídas a ajustes pobres por regressão ou modelos de ajustes impróprios da curva de retenção, medições errôneas de dados experimentais, solos com porosidade heterogênea, entre

\footnotetext{
1 UNESP (alfaro@rc.unesp.br).
}

2 UNESP (chang@rc.unesp.br). 
outros. Segundo Mallants et al. (1996), vários autores (SMETTEN e KIRKBY, 1990; OTHMER et al., 1991; DURNER, 1994) mostraram erros de mais de $30 \%$ na previsão de $\mathrm{k}(\theta)$, quando baseados em modelos de curva de retenção unimodais para solo com distribuição de tamanhos de poros multimodal. Em contrapartida, Othmer et al. (1991) obtiveram melhores resultados da função condutividade hidráulica empregando modelos bimodais no ajuste de curvas de retenção.

Segundo Alfaro Soto et al. (2008), muitos solos brasileiros podem mostrar comportamento multimodal na distribuição do tamanho de poros. No entanto, não há registros de estudos de condutividade hidráulica em solo não saturado com sistemas de poros multimodais; presume-se, assim, que os estudos de transporte de água ou contaminantes (seja em solo saturado ou não saturado), realizados no âmbito nacional, não levem em consideração tal característica.

Tendo em vista que em estudos de áreas contaminadas a avaliação das características hidráulicas é feita com base na permeabilidade relativa (relacionando as condutividades hidráulicas não saturada e saturada do meio poroso), este trabalho busca avaliar o comportamento dessa propriedade em alguns solos brasileiros que apresentam distribuição de tamanhos de poros com comportamento bimodal. Esse comportamento é estimado por métodos indiretos, que se valem de curvas de retenção para representar o tipo de distribuição. Os resultados foram comparados com estimativas de permeabilidade relativa obtidos por modelos tradicionais, com a finalidade de evidenciar o erro associado. Pretende-se, com isso, estimular o emprego de novos modelos que possam ser aplicados satisfatoriamente na solução de problemas mecânicos e hidráulicos de solos (ou de meio porosos) que apresentem esta peculiaridade.

\section{MODELOS TEÓRICOS E EXPERIMEN- TAIS DAS PROPRIEDADES HIDRÁULICAS DE SOLOS}

Este item apresenta os modelos empregados na caracterização das propriedades hidráulicas no presente estudo, com a finalidade de avaliar a adequabilidade de sua utilização em solos que apresentam distribuição de tamanhos de poros multimodais, comumente encontrados no Brasil. Adicionalmente, são apresentadas as características dos solos utilizados nesta análise.
Modelos tradicionais para descrição das propriedades hidráulicas em solos não saturados Curva de retenção de água no solo

Van Genchuten (1980) desenvolveu uma equação que relaciona sucção ao grau de saturação e se destina ao ajuste dos dados experimentais de curvas de retenção de água no solo. Em termos de saturação efetiva, esta equação é dada por:

$S_{e}=\left(1+(\alpha h)^{n}\right)^{-m}$

onde:

$S_{e}=\left(S-S_{r}\right) /\left(1-S_{r}\right)$

Na equação (1), os parâmetros $\alpha\left[\mathrm{L}^{-1}\right], n[-$ ] e $m[-]$ correspondem aos parâmetros de ajuste e $h$ [L] representa a sucção. Segundo van Genuchten (1980), $\alpha$ e $n$ relacionam-se com a pressão de entrada de ar e com a distribuição de tamanho dos poros, respectivamente. Por outro lado, $m$ pode ser relacionado com $n$ : segundo Mualem (1976), $m=1-1 / n$, e para Burdine (1953), $m=1$ $2 / n$. Na equação (2), $S_{e}, S$ e $S_{r}$ correspondem à saturação efetiva, saturação para uma determinada sucção e saturação irredutível, respectivamente.

São muitas as equações destinadas à determinação da curva de retenção da água no solo, a maioria de caráter empírico, e apresentam diferentes graus de sucesso em relação à sua aplicabilidade (BACCHI, 1993).

A maioria dos modelos existentes na literatura especializada pode ser utilizada para solos cuja distribuição de tamanhos de poros é unimodal, ou seja, esses modelos retratam satisfatoriamente as características das curvas de retenção de água em solos que não apresentem significativa heterogeneidade em relação a sua porosidade.

A distribuição dos poros pode ser avaliada a partir da curva de retenção. Segundo Stoicescu et al. (1996), isso é possível pela derivação da equação (1) em relação à sucção. Essa equação é dada por:

$f(h)_{u}=\frac{m n \alpha(\alpha h)^{n-1}}{\left(1+(\alpha h)^{n}\right)^{m+1}}$

onde $f(h)_{\mathrm{u}}$ representa a distribuição de tamanho de poros unimodal em função da sucção.

\section{Permeabilidade relativa da água}

Baseado nas equações de Burdine (1953) e Mualem (1976), van Genuchten (1980) desenvolveu equações para previsão da permeabilidade relativa da água. $\mathrm{O}$ modelo estatístico de Mualem 
(1976), empregado para obtenção da função permeabilidade relativa unimodal de fluidos molhantes, com base na curva de retenção, pode ser expresso da forma seguinte:

$k_{r w}\left(S_{e}\right)=S_{e}^{0,5} \cdot\left[\frac{\int_{S_{r}}^{S_{e}} \frac{d S}{h}}{\int_{S_{r}}^{1} \frac{d S}{h}}\right]^{2}$

onde $k_{r w}=k\left(S_{e}\right) / k_{s a t}$ corresponde à permeabilidade relativa; $k\left(S_{e}\right)$ representa a função de condutividade hidráulica de solo não saturado e $k_{\text {sat }}$, é a condutividade hidráulica de solo saturado.

A obtenção de $k_{r w}$ para a distribuição do tamanho dos poros unimodal, com base na curva de retenção, consiste em isolar $h$ (sucção) da equação (1) e introduzi-la na equação (4). Dessa forma, ao resolver a integração tem-se como resultado a equação de van Genuchten-Mualem, dada por:

$k_{r w}\left(S_{e}\right)=S_{e}^{0,5}\left(1-\left(1-S_{e}^{1 / m}\right)^{m}\right)^{2}$

A equação (4) também pode ser utilizada para obtenção da permeabilidade relativa (similar à equação 5), em solos que apresentem distribuição de tamanhos de poros multimodal, desde que seja utilizado um modelo adequado de curva de retenção, tal como será visto a seguir.

\section{Modelos multimodais para descrição das pro-} priedades hidráulicas em solos não saturados Curva de retenção de água no solo

Segundo Mallants et al. (1997), a heterogeneidade de um sistema de poros deve-se a uma distribuição granulométrica específica ou à formação de porosidade secundária relacionada a processos genéticos diversos, tais como agregação física, processos biológicos, solos glaciais (morenas), processos de solifluxão de solos, entre outros.

A esse respeito, Alfaro Soto et al. (2008) mostraram que vários solos do estado de São Paulo apresentam descontinuidades no escalonamento de tamanhos dos poros, devido à estruturação, em que a macroporosidade é provavelmente ocasionada pela agregação de partículas de menor tamanho ou por processos biológicos, produzindo distribuições de tamanhos de poros bimodal ou trimodal. A Figura 1 mostra curvas de retenção (sucção versus umidade volumétrica) obtidas para alguns solos do interior do estado de São Paulo, e a Figura 2 apresenta as correspondentes distribui- ções do tamanho de poros para esses solos, calculados pela equação (3).

As curvas de retenção da figura 1 mostram comportamentos característicos de solos com distribuições de tamanho de poros multimodais, apresentando mais de dois vértices ao longo da curva. Assim, foram necessários no mínimo dois ajustes da curva de retenção para caracterizar toda a faixa de umidade estudada. Esses solos apresentam duas modas, exceto o solo 1 que se mostrou trimodal (Figura 2).

A distribuição de tamanhos de poros bimodal não é observada somente em materiais não consolidados, mas também em rochas. Segundo Gerke e van Genuchten (1993), tal bimodalidade está associada à macroporosidade ou às fraturas da rocha,e aos poros de menores tamanhos da matriz rochosa.

Em vista da heterogeneidade no tamanho dos poros do solo, a curva de retenção pode ser modelada, segundo Durner (1994), por meio da superposição linear de subcurvas provenientes do modelo de van Genuchten (1980), que é expresso por:

$$
S_{e}=\sum_{i=1}^{k} w_{i}\left(1+\left(\alpha_{i} h\right)^{n_{i}}\right)^{-m_{i}}
$$

onde $k$ representa o número de subsistemas que formam a distribuição total de poros e $w_{i}$ são os fatores designados para os pesos das subcurvas que variam entre 0 e 1 , isto é, $\Sigma w_{i}=1$. Da mesma forma que no modelo unimodal, os parâmetros das subcurvas $\alpha_{i}, n_{i}$ e $m_{i}$ estão sujeitos aos seguintes condicionantes: $\alpha_{i}>0, n_{i}>1$ e $m_{i}>1$.

\section{Permeabilidade relativa da água}

Segundo Šimůnek et al. (2003), a permeabilidade relativa para um sistema multimodal de poros pode ser obtida pela superposição linear das funções de cada região em particular. A partir das equações (6) e (4) pode-se obter a seguinte expressão para um sistema de poros multimodal:

$$
k_{r w}\left(S_{e}\right)=\left(\sum_{i=1}^{k} w_{i} S_{e}\right)^{0,5} \frac{\left(\sum_{i=1}^{k} w_{i} \alpha_{i}\left(1-\left(1-S_{e}{ }^{1 / m}\right)^{m}\right)\right)^{2}}{\left(\sum_{i=1}^{k} w_{i} \alpha_{i}\right)^{2}}
$$

Adicionalmente, para verificação da qualidade do ajuste foram realizadas análises para determinação do erro quadrático médio (EQM). Este indicador do ajuste pode ser obtido por:

$$
E Q M=\sqrt{\frac{\left[\log \left(X_{\text {med }}\right)-\log \left(X_{\text {prev }}\right)\right]^{2}}{n-1}}
$$


onde $X_{\text {med }}$ e $X_{\text {prev }}$ representam os parâmetros hidráulicos (saturação efetiva ou permeabilidade relativa da água) medido e previsto, respectivamente, e $n$ representa o número de amostras. $\mathrm{O}$ emprego do $\log \left(X_{i}\right)$ deve-se ao fato que, segundo métodos estatísticos (método de Kolmorogov-
Smirnof), as amostras seguem uma distribuição $\log$-normal.

Cabe ressaltar que valores de EQM próximos a zero significam melhores previsões. Em contrapartida, valores contrários são indicadores de maior desvio em relação aos valores medidos.

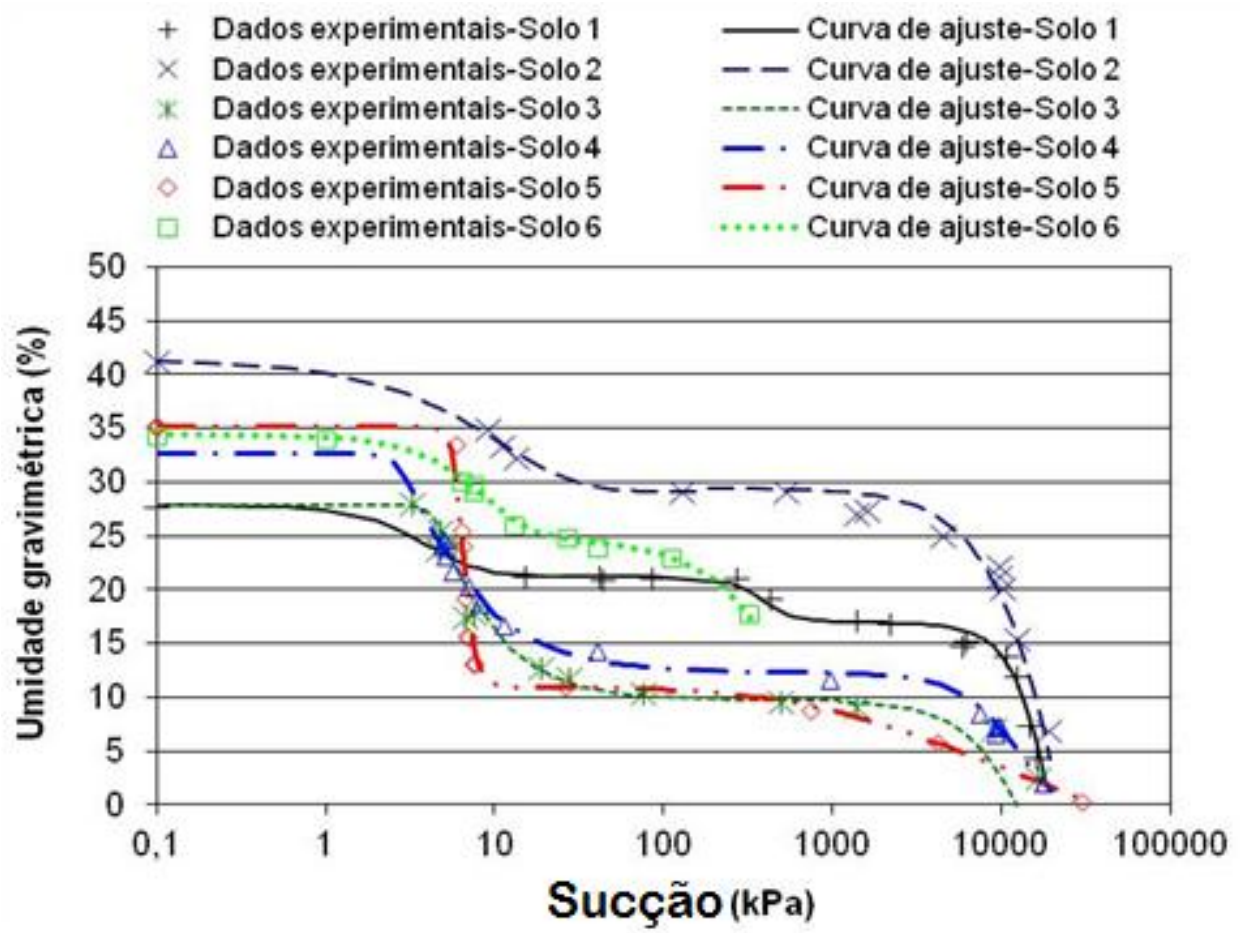

Figura 1 - Curvas de retenção de água no solo para alguns solos do interior do estado de São Paulo (ALFARO SOTO et al., 2008).

Figure 1 - Curvas de retenção de água no solo para alguns solos do interior do estado de São Paulo (ALFARO SOTO et al., 2008).

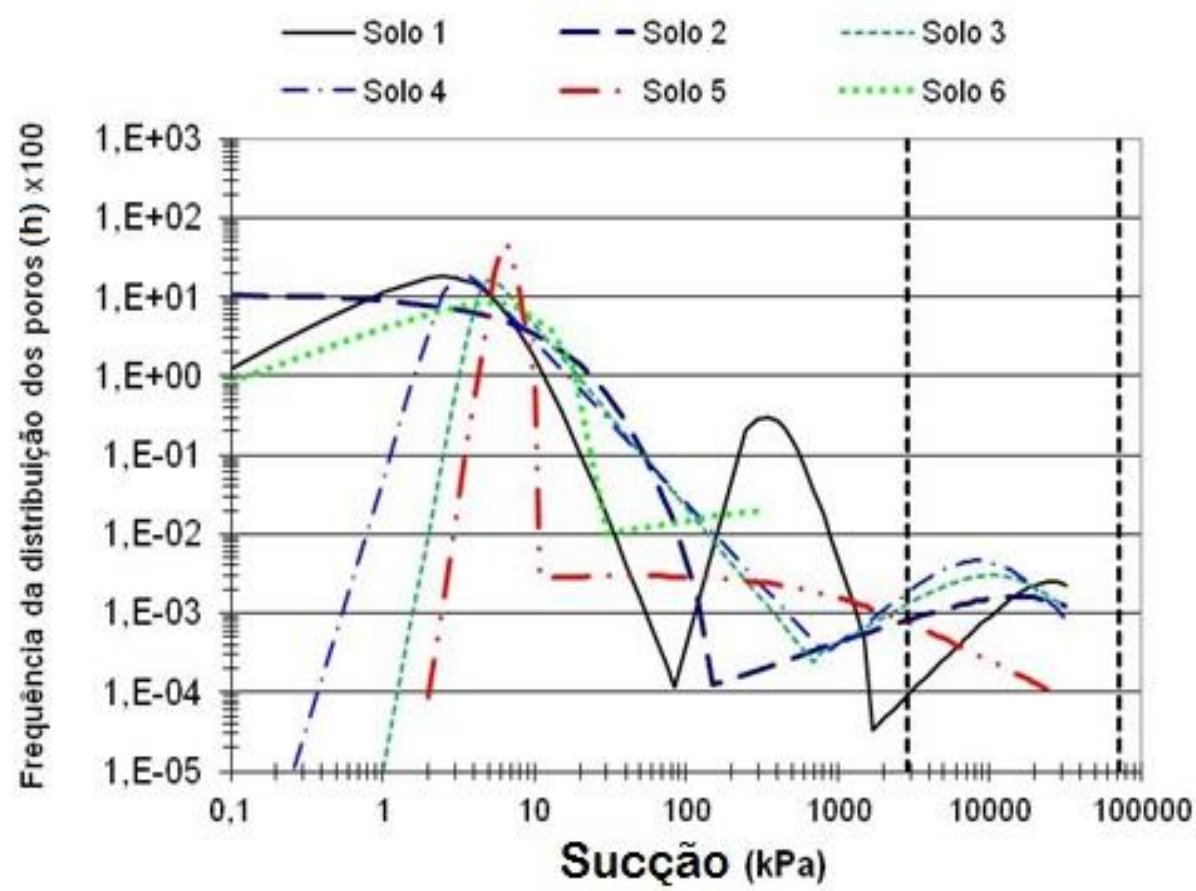

Figura 2 - Distribuição de poros de alguns solos do interior do estado de São Paulo (ALFARO SOTO et al., 2008).

Figure 2 - Distribuição de poros de alguns solos do interior do estado de São Paulo (ALFARO SOTO et al., 2008). 


\section{Caracterização petrofísica dos solos estudados}

Para avaliação do comportamento de curvas de retenção e permeabilidade relativa bimodal foram utilizados seis tipos de solos. O solo A é um material não consolidado proveniente da cidade de Rio Claro-SP, o solo B, da cidade de Itirapina-SP, o solo C, da cidade de Lins-SP, o solo D, da cidade de Londrina-PR (MIGUEL et al., 2006), e os solos E e F, da cidade de São Carlos-SP (GRACIOSA et al., 2008).

Os testes para determinação da curva de retenção foram realizados em laboratório, para a maioria dos solos, tendo sido utilizado em todos o processo de secagem. No entanto, os testes dos solos A, B e C foram realizados pelo método do papel de filtro (MARINHO, 1994), enquanto do solo D, pelo método da câmara de pressão (ASTM, 2008). Os únicos testes conduzidos em campo foram os dos solos E e F, cujas medidas foram realizados em perfis de solo, com auxílio de aparelhos tais como sondas de nêutrons e tensiômetros.

Cabe ressaltar que as informações sobre os testes aqui apresentados são provenientes da literatura nacional especializada e não contam com resultados experimentais de permeabilidade relativa da água para solos com poros com distribuição de tamanhos multimodais. A determinação da permeabilidade relativa unimodal e bimodal foi obtida, neste trabalho por métodos indiretos, com a finalidade única de mostrar a discrepância dos resultados para um determinado valor de sucção.

\section{RESULTADOS E DISCUSSÃO}

Avaliação de curvas de retenção nos solos estudados

Os solos empregados apresentam texturas que variam de arenosa a argilosa. Tal variação é desejável, pois permite observar o comportamento das características da curva de retenção e de permeabilidade relativa em solos com texturas variadas e porosidades heterogêneas. Na Tabela 1 são apresentadas algumas características geotécnicas desses materiais.

Tabela 1 - Características geotécnicas dos solos estudados Table 1 - Características geotécnicas dos solos estudados

\begin{tabular}{|c|c|c|c|c|c|c|c|}
\hline \multirow[t]{2}{*}{ Parâmetros } & \multirow[t]{2}{*}{ Unidades } & \multicolumn{6}{|c|}{ Solo } \\
\hline & & $\mathrm{A}$ & $\mathrm{B}$ & $\mathrm{C}$ & $\mathrm{D}$ & $\mathrm{E}$ & $\mathrm{F}$ \\
\hline Pedregulho & $(\%)$ & 0,0 & 1,4 & 0,1 & 8,8 & 0,0 & 0,0 \\
\hline Areia & $(\%)$ & 69,0 & 79,6 & 69,9 & 68,9 & 55,0 & 7,0 \\
\hline Silte & $(\%)$ & 8,0 & 1,6 & 5,0 & 5,8 & 28,8 & 12,0 \\
\hline Argila & $(\%)$ & 23,0 & 17,4 & 25,0 & 16,5 & 16,2 & 81,0 \\
\hline$\square$ & $\left(\mathrm{g} . \mathrm{cm}^{-3}\right)$ & 2,58 & 2,67 & 2,70 & 2,65 & 2,89 & 3,06 \\
\hline$\square_{\mathrm{d}}$ & $\left(\mathrm{g} . \mathrm{cm}^{-3}\right)$ & 1,50 & 1,38 & 1,69 & 1,70 & 1,36 & 1,40 \\
\hline e & $(-)$ & 0,42 & 0,48 & $\begin{array}{c}0,37 \\
\text { Areia }\end{array}$ & $\begin{array}{c}0,36 \\
\text { Areia fina }\end{array}$ & 0,53 & 0,54 \\
\hline Textura (ABNT) & $(-)$ & $\begin{array}{l}\text { Areia mé- } \\
\text { dia argilosa }\end{array}$ & $\begin{array}{l}\text { Areia mé- } \\
\text { dia argilosa }\end{array}$ & $\begin{array}{c}\text { média a } \\
\text { fina argi- } \\
\text { losa }\end{array}$ & $\begin{array}{l}\text { argilosa } \\
\text { com pe- } \\
\text { dregulho }\end{array}$ & $\begin{array}{c}\text { Areia fina } \\
\text { siltosa }\end{array}$ & $\begin{array}{l}\text { Argila } \\
\text { siltosa }\end{array}$ \\
\hline
\end{tabular}

$\rho$ e $\rho_{\mathrm{d}}$ : massa específica dos sólidos e do solo, respectivamente; e: índice de vazios.

Adicionalmente, a Figura 3 mostra os resultados experimentais e ajustados (LEVENBERG-MARQUARDT; MARQUARDT, 1963) da curva de retenção, para os seis solos estudados, utilizando-se os modelos expressos pelas equações (1) e (6) para sistemas porosos que apresentam comportamento unimodal e bimodal, respectivamente, considerando-se uma ampla faixa de sucção. A Tabela 2 apresenta os parâmetros de ajuste obtidos e os coeficientes de determinação $\left(R^{2}\right)$, enquanto a Tabela 3 sintetiza os resultados de EQM obtidos para cada solo.

Da Figura 3 pode-se inferir que as curvas em geral seguem um comportamento típico de solos arenosos e argilosos, de acordo com sua textura (Tabela 1). Porém, é evidente a forma escalonada dos dados experimentais e a dificuldade de ajuste adequado utilizando apenas o modelo unimodal (Equação 1), enquanto que o modelo multimodal (Equação 6) apresenta melhor ajuste aos dados experimentais. 


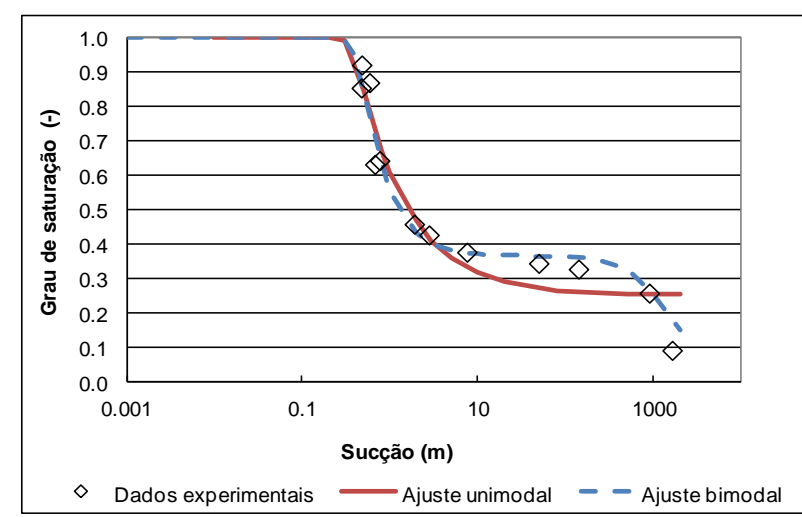

(a)

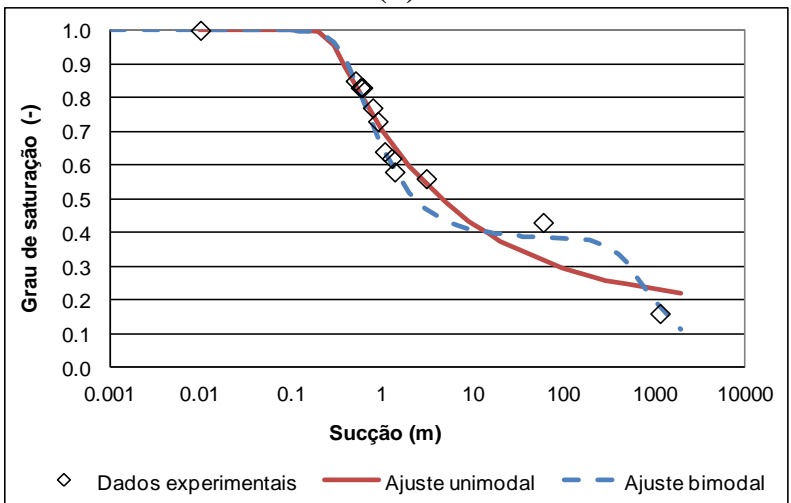

(c)

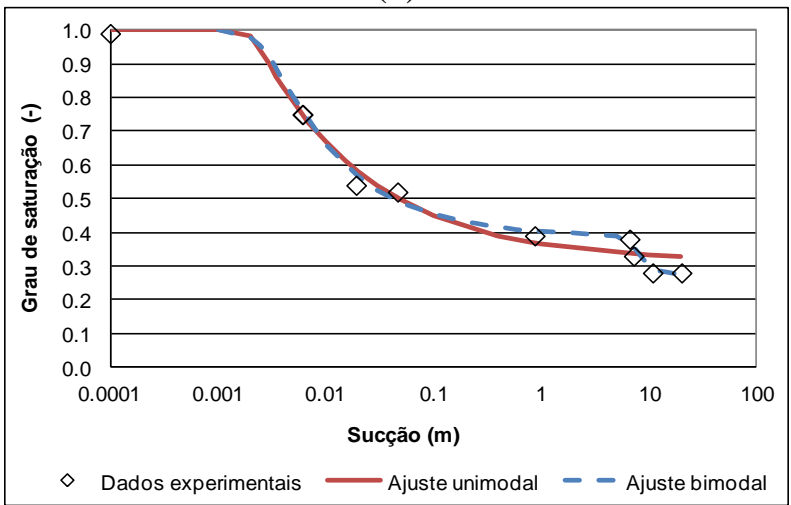

(e)

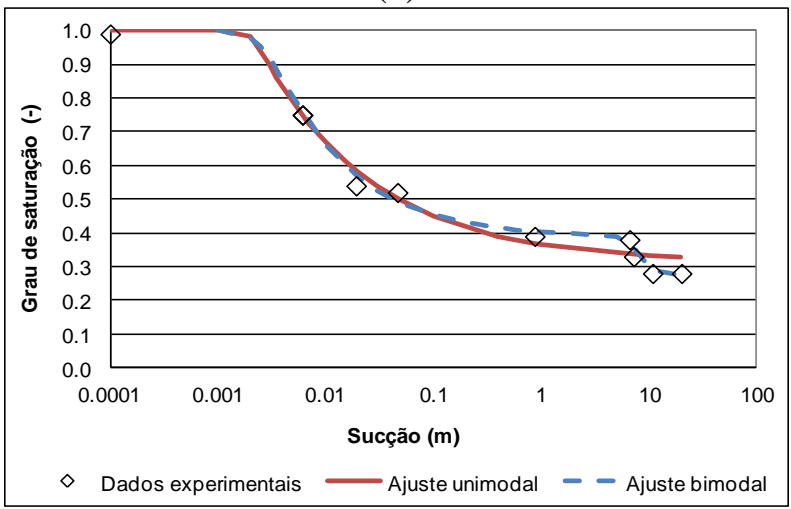

(e)

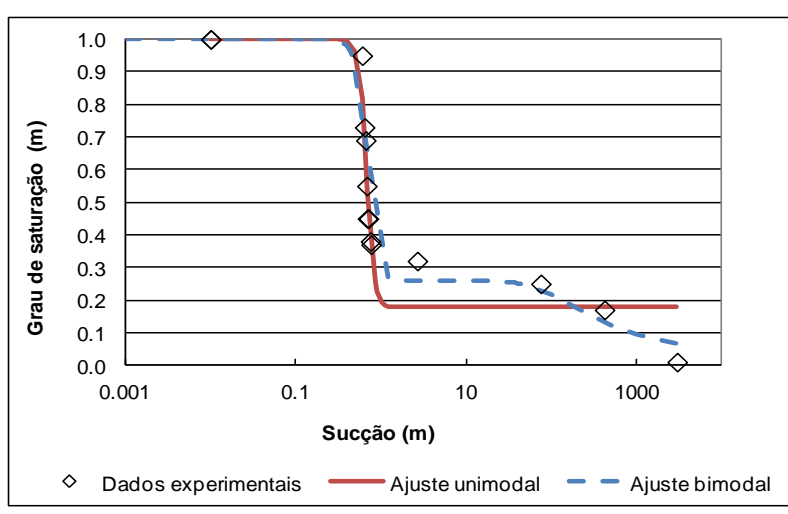

(b)

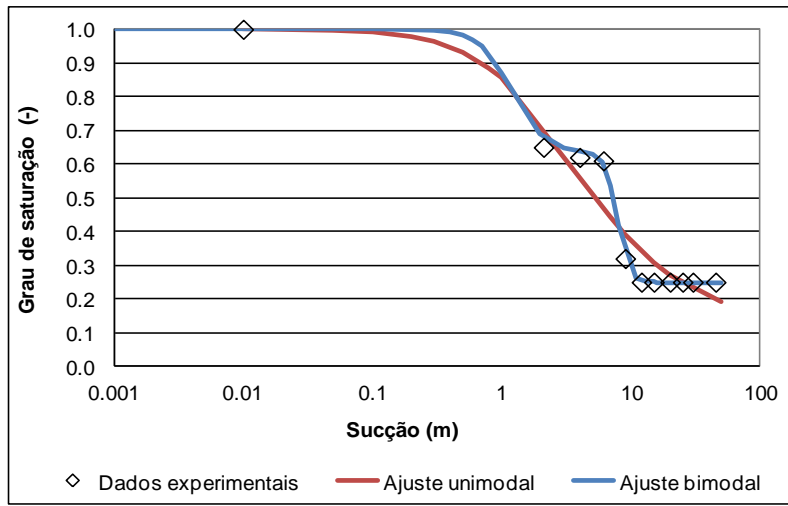

(d)

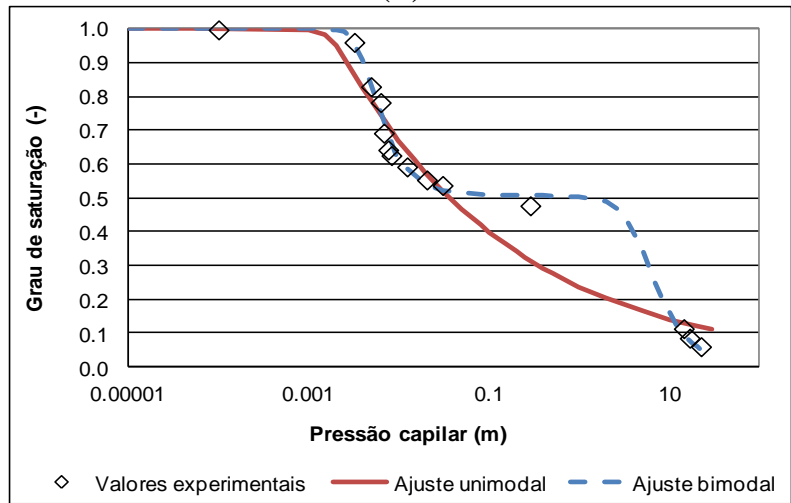

(f)

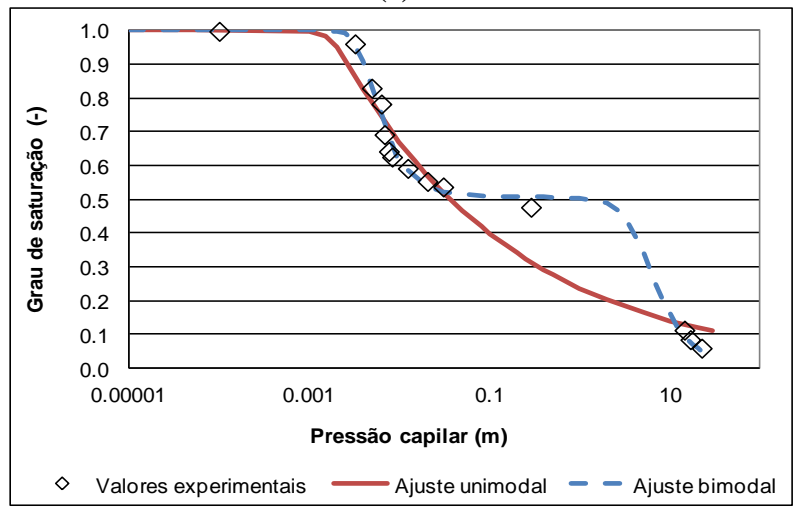

(f)

Figura 3 - Valores experimentais e ajustados de curvas retenção de água no solo uni e bimodal para os solos das cidades de: (a) Rio Claro-SP; (b) Itirapina-SP; (c) Lins-SP; (d) Londrina-PR (MIGUEL et al., 2006); (e, f) São Carlos-SP (GRACIOSA et al., 2008)

Figure 3 - Valores experimentais e ajustados de curvas retenção de água no solo uni e bimodal para os solos das cidades de: (a) Rio Claro-SP; (b) Itirapina-SP; (c) Lins-SP; (d) Londrina-PR (MIGUEL et al., 2006); (e, f) São Carlos-SP (GRACIOSA et al., 2008) 
Tabela 2 - Parâmetros de ajuste por regressão às curvas de retenção dos solos da Figura 3

Table 2 - Parâmetros de ajuste por regressão às curvas de retenção dos solos da Figura 3

\begin{tabular}{|c|c|c|c|c|c|c|c|c|}
\hline \multirow[t]{2}{*}{ Ajuste } & \multirow[t]{2}{*}{ Parâmetros } & \multirow[t]{2}{*}{ Und } & \multicolumn{6}{|c|}{ Solo } \\
\hline & & & $\mathrm{A}$ & $\mathrm{B}$ & $\mathrm{C}$ & D & $\mathrm{E}$ & $\mathrm{F}$ \\
\hline \multirow{5}{*}{ Uni-modal } & $\alpha$ & $\left(\mathrm{m}^{-1}\right)$ & 2,71 & 1,47 & 590,59 & 3,92 & 491,79 & 0,39 \\
\hline & $\mathrm{N}$ & $(-)$ & 9,68 & 10,00 & 5,57 & 9,99 & 10,00 & 1,26 \\
\hline & M & $(-)$ & 0,08 & 1,00 & 0,04 & 0,03 & 0,04 & 0,67 \\
\hline & $\mathrm{S}_{\mathrm{r}}$ & $(-)$ & 0,25 & 0,18 & 0,0001 & 0,18 & 0,31 & 0,12 \\
\hline & $\mathrm{R}^{2}$ & $(-)$ & 0,75 & 0,49 & 0,89 & 0,87 & 0,95 & 0,92 \\
\hline \multirow{9}{*}{ Bi-modal } & $\mathrm{w}_{1}$ & $(-)$ & 0,63 & 0,74 & 0,50 & 0,62 & 0,84 & 0,50 \\
\hline & $\alpha_{1}$ & $\left(\mathrm{~m}^{-1}\right)$ & 2,17 & 1,53 & 245,55 & 2,22 & 379,63 & 0,13 \\
\hline & $\alpha_{2}$ & $\left(\mathrm{~m}^{-1}\right)$ & 0,001 & 0,015 & 0,204 & 0,002 & 0,13 & 0,96 \\
\hline & $\mathrm{n}_{1}$ & $(-)$ & 6,55 & 7,48 & 5,52 & 3,55 & 4,49 & 9,99 \\
\hline & $\mathrm{n}_{2}$ & $(-)$ & 2,11 & 2,46 & 3,04 & 2,96 & 7,06 & 3,67 \\
\hline & $\mathrm{m}_{1}$ & $(-)$ & 0,24 & 1,00 & 0,31 & 0,29 & 0,14 & 1,00 \\
\hline & $\mathrm{m}_{2}$ & $(-)$ & 0,53 & 0,15 & 0,51 & 0,30 & 0,70 & 0,70 \\
\hline & $\mathrm{S}_{\mathrm{r}}$ & $(-)$ & 0,0001 & 0,0001 & 0,0022 & 0,0001 & 0,2716 & 0,2487 \\
\hline & $\mathrm{R}^{2}$ & $(-)$ & 0,896 & 0,781 & 0,971 & 0,998 & 0,989 & 0,991 \\
\hline
\end{tabular}

Tabela 3 - Erros quadráticos médios entre dados experimentais e ajustados pelos modelos (1) e (6) para obtenção da curva de retenção dos solos estudados

Table 3 - Erros quadráticos médios entre dados experimentais e ajustados pelos modelos (1) e (6) para obtenção da curva de retenção dos solos estudados

\begin{tabular}{llcc}
\hline Solos & Descrição & $\begin{array}{c}\text { Unimodal } \\
\text { Eq. (1) }\end{array}$ & $\begin{array}{c}\text { Bimodal } \\
\text { Eq.(6) }\end{array}$ \\
\hline a & Rio Claro-SP & 0,14 & 0,09 \\
b & Itirapina - SP & 0,43 & 0,31 \\
c & Lins - SP & 0,09 & 0,05 \\
d & Londrina-PR (Miguel et al., 2006) & 0,11 & 0,01 \\
e & São Carlos-SP (Graciosa et al., 2008). & 0,06 & 0,03 \\
$\mathrm{f}$ & São Carlos-SP (Graciosa et al., 2008). & 0,14 & 0,05 \\
\hline
\end{tabular}

Os resultados de $\mathrm{R}^{2}$, provenientes do modelo multimodal (Equação 6), mostram a melhor aderência das curvas de ajuste aos dados experimentais, pois em todos os casos os coeficientes $\mathrm{R}^{2}$ são mais próximos a 1 . Por outro lado, os valores de EQM da Tabela 3 confirmam esta tendência, uma vez que se apresentam mais baixos em relação aos provenientes do modelo unimodal (Equação 1). Ainda, a partir desses resultados pode-se deduzir que os melhores ajustes bimodais foram encontrados para os solos C, D, E e F.

\section{Avaliação da distribuição do tamanho de poros nos solos estudados}

A avaliação das distribuições de tamanhos de poros, unimodal e bimodal, foi feita a partir das curvas ajustadas de retenção. Para a determinação da frequência de distribuição unimodal do tamanho dos poros foi utilizada a equação (3), proposta por Stoicescu et al. (1996). Com base no procedimento utilizado por esses autores, foi desenvolvida, neste trabalho, a formulação para a frequência de distribuição bimodal, resultando:

$$
f(h)_{b}=\frac{w_{1} m_{1} n_{1} \alpha_{1}\left(\alpha_{1} h\right)^{n_{1}-1}}{\left(1+\left(\alpha_{1} h\right)^{n_{1}}\right)^{m_{1}+1}}+\frac{w_{2} m_{2} n_{2} \alpha_{2}\left(\alpha_{2} h\right)^{n_{2}-1}}{\left(1+\left(\alpha_{2} h\right)^{n_{2}}\right)^{m_{2}+1}}
$$

onde $f(h)_{\mathrm{b}}$ representa a distribuição dos poros bimodal e os parâmetros $w_{i}, m_{i}, n_{i}, \alpha_{i}$ são os mesmos da equação (6).

A Figura 4 ilustra os resultados da sucção versus frequência de distribuição dos poros para cada solo estudado e que cada modelo reproduz. Tendo em vista a relação direta entre curva de retenção e distribuição do tamanho dos poros e que o ajuste de curva de retenção pela equação (6) é, em todos os casos, mais coerente com os dados experimentais, pode-se afirmar que os solos estudados possuem distribuições de tamanho de poros bimodais. Como mencionado em Alfaro Soto et al. (2008), os picos identificam o início de escalonamentos de tamanho de poros que começam, aproximadamente, nas sucções correspondentes à pressão de entrada de ar (pressão menor) e de saturação irredutível (pressão maior), respectivamente. 


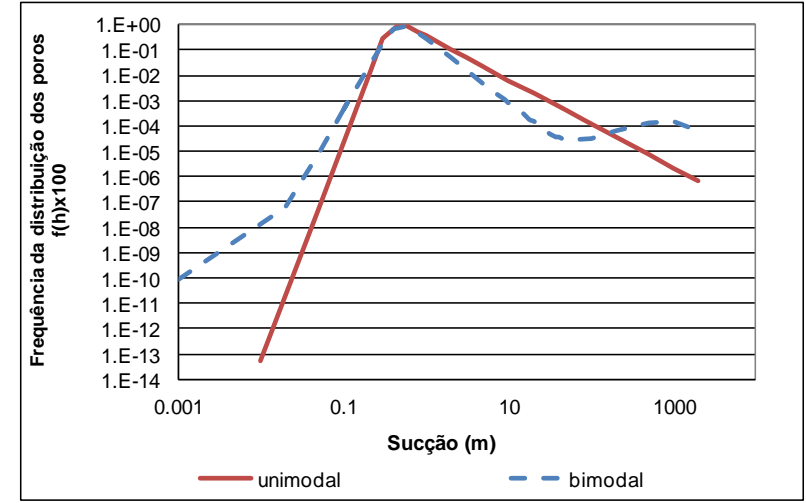

(a)

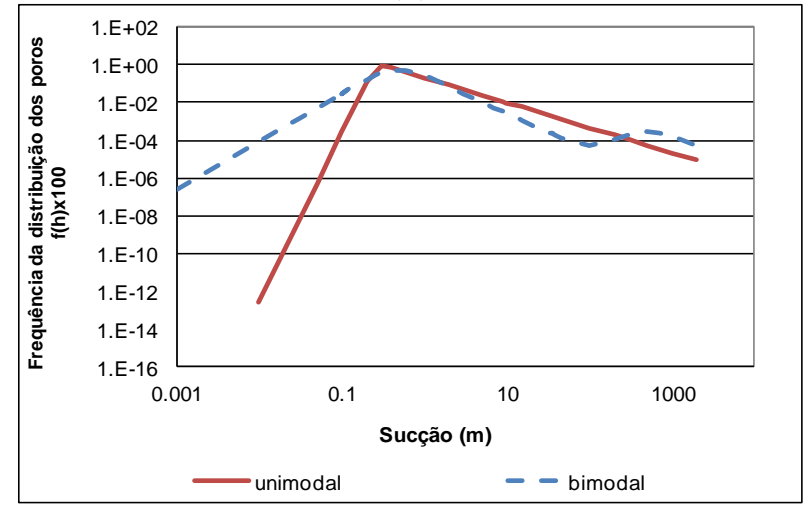

(c)

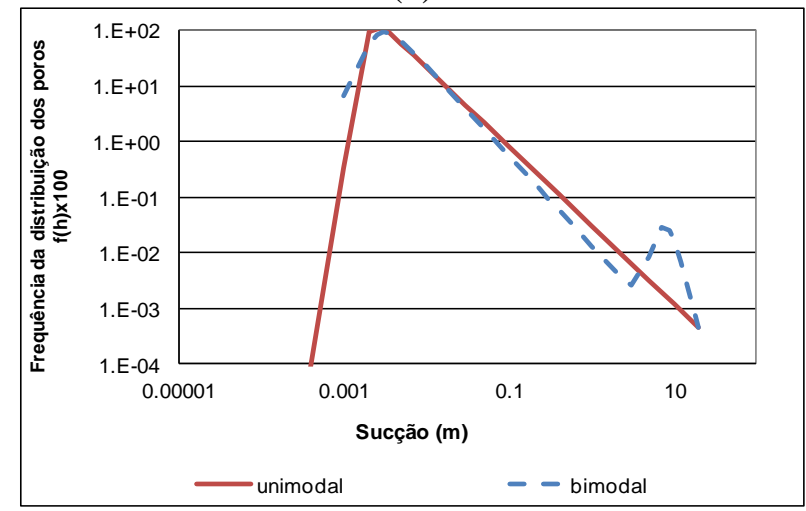

(e)

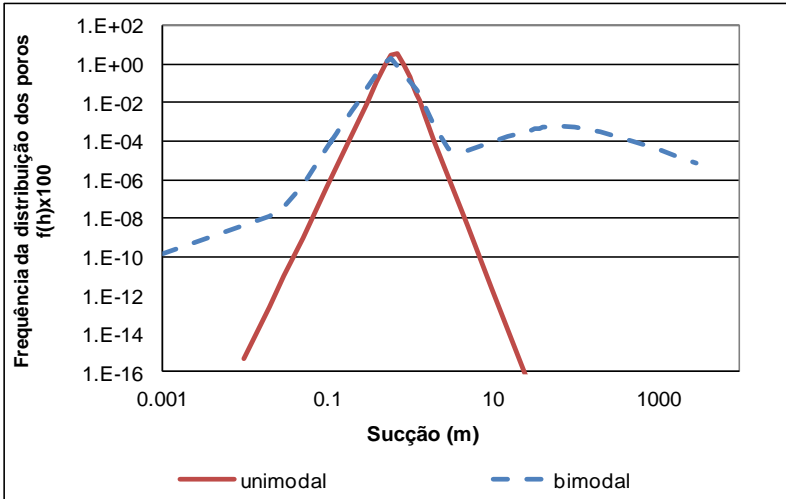

(b)

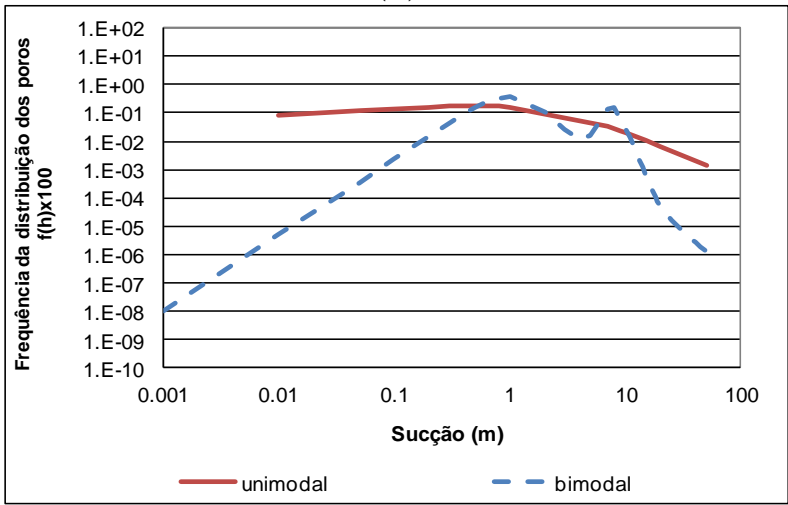

(d)

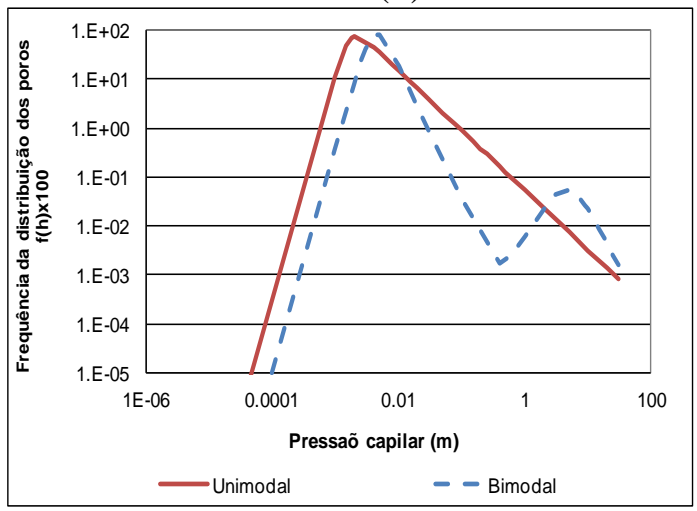

(f)

Figura 4 - Sucção versus frequência da distribuição dos poros para os solos das cidades de: (a) Rio ClaroSP; (b) Itirapina-SP; (c) Lins-SP; (d) Londrina- PR (MIGUEL et al., 2006); (e, f) São Carlos-SP (GRACIOSA et al., 2008)

Figure 4 - Sucção versus frequência da distribuição dos poros para os solos das cidades de: (a) Rio ClaroSP; (b) Itirapina-SP; (c) Lins-SP; (d) Londrina- PR (MIGUEL et al., 2006); (e, f) São Carlos-SP (GRACIOSA et al., 2008)

Esse fato se relaciona com a forma escalonada da curva de retenção, bem como às dificuldades para o ajuste que ocorrem, em geral, na faixa intermediária da sucção matricial (ou tamanho de poros intermediários). De acordo com Durner (1994), o problema se deve possivelmente a processos de agregação de partículas de menor tamanho. A agregação pode ocorrer por agentes cimentantes (minerais de argila, matéria orgânica, óxidos de ferro e alumínio), formando coloides, poros secundários e poros maiores. Para Othmer et al. (1991), nesses sistemas bimodais, nos poros maiores, denominados poros interagregados, o movimento de água no solo é governado pelo potencial gravitacional. Por outro lado, nos poros de menor tamanho, denominados intra-agregados, a água se movimenta graças ao potencial matricial (ALFARO SOTO et al., 2008). 


\section{Avaliação da permeabilidade relativa nos solos estudados}

Para avaliações dos modelos de permeabilidade relativa unimodal e multimodal foram empregadas as equações decorrentes do modelo de van Genuchten-Mualem (van GENUCHTEN, 1980) e Durner (1994)-Mualem Šimůnek et al., 2003), dadas pelas equações (5) e (7), respectivamente.

Os parâmetros necessários para essas formulações são os mesmos utilizados para o ajuste das curvas de retenção dos solos da Tabela 2. A Figura 5 mostra os resultados da permeabilidade relativa unimodal e multimodal versus o logaritmo decimal da sucção, expressa em centímetros de água $(\mathrm{pF})$ para os solos estudados.

Em todos os casos, resulta evidente a bimodalidade na forma da curva de permeabilidade relativa quando utilizado o modelo (7). De acordo com o observado, pode-se inferir que o ajuste pelo modelo unimodal (Equação 1) pode subestimar ou superestimar (Figura 5d) o valor da permeabilidade relativa.

Para esses solos, as diferenças da permeabilidade relativa unimodal e bimodal se apresentam progressivas com acréscimos da sucção, podendo fazer diferir a condutividade hidráulica não saturada em mais de duas ordens de grandeza.

Outro fato a observar é que o baixo ajuste da curva de retenção no modelo unimodal, principalmente a partir da metade até o final da curva de dados experimentais (onde ocorre geralmente a forma escalonada), compromete os resultados de previsão da permeabilidade relativa. Dessa forma, pode-se deduzir que os parâmetros " $m$ " e " $\alpha$ " da curva de retenção da água no solo (Equação 1) apresentam grande influência nos resultados da permeabilidade relativa (Equação 5). Sendo assim, é recomendável a medição desses parâmetros nesses tipos de solos. Caso contrário, a adoção desses parâmetros com base na textura do solo proveniente da bibliografia especializada deverá ser criteriosa, pois não necessariamente leva em consideração a estruturação de solos característicos de algumas regiões do Brasil.

Infelizmente, no Brasil são escassos (ou não foram conduzidos visando observar este fenômeno) os resultados de testes de condutividade hidráulica em solos com distribuição de poros mutimodal, porém as curvas de retenção com características peculiares, já observadas por diversos autores (OLIVEIRA et al., 2004; LOUGONDUARTE et al., 2004; FEUERHARMEL et al., 2004, ALFARO SOTO et al., 2008, entre outros), não deixam dúvidas de que este fenômeno ocorra.

Como já comentado, não se têm dados experimentais de permeabilidade relativa, o que impossibilita uma avaliação direta da eficácia dos resultados fornecidos pelos modelos uni e multimodal. No entanto, foram avaliados os desvios pela utilização do modelo unimodal assumindo-se a exequibilidade do modelo (7), já demonstrada por outros autores (MALLANTS et al., 1997; ŚIMŮNEK et al., 2003, entre outros). A Tabela 4 reúne os resultados de EQM obtidos para todos os solos estudados.

Tabela 4 - Erros quadráticos médios para os dados previstos pelo modelo unimodal (5) para obtenção da permeabilidade relativa dos solos estudados

Table 4 - Erros quadráticos médios para os dados previstos pelo modelo unimodal (5) para obtenção da permeabilidade relativa dos solos estudados

\begin{tabular}{llc}
\hline Solos & Descrição & Unimodal \\
Eq.(5) \\
\hline a & Rio Claro-SP & 16,4 \\
b & Itirapina - SP & 10,4 \\
c & Lins - SP & 6,7 \\
d & Londrina-PR (Miguel et al., 2006) & 1,2 \\
e & São Carlos-SP (Graciosa et al., 2008). & 0,4 \\
f & São Carlos-SP (Graciosa et al., 2008). & 1,0 \\
\hline
\end{tabular}




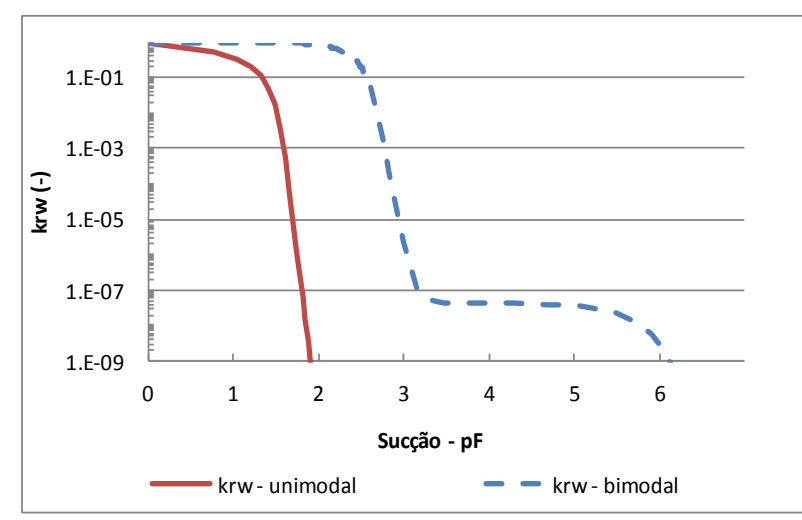

(a)

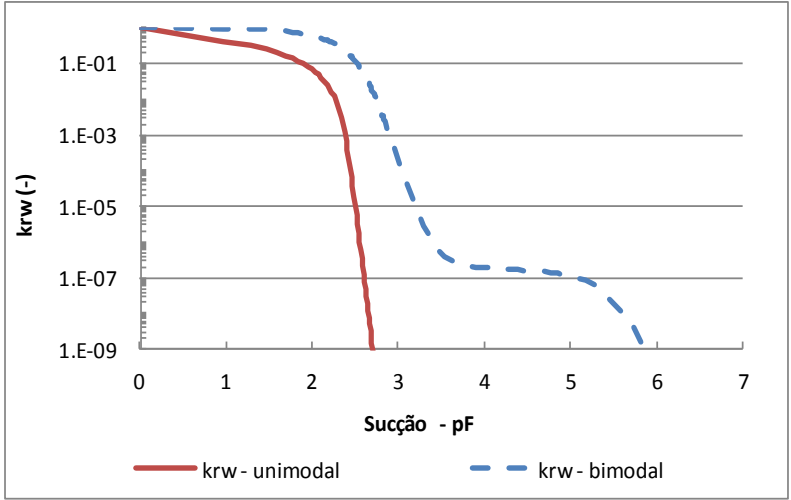

(c)

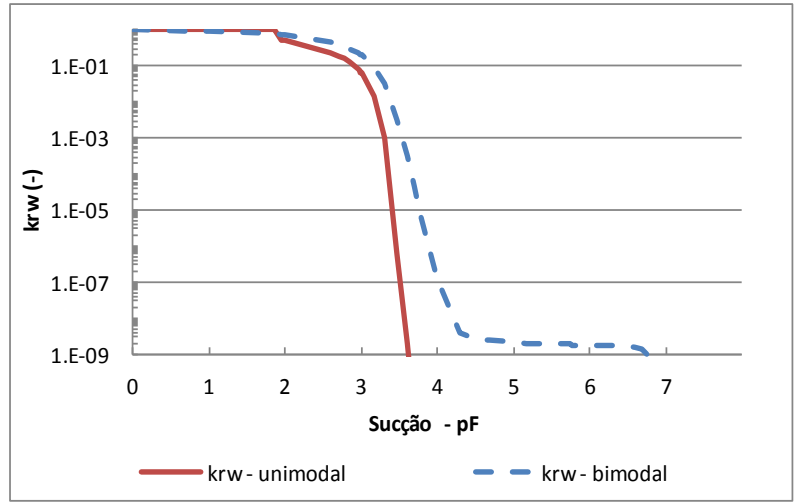

(e)

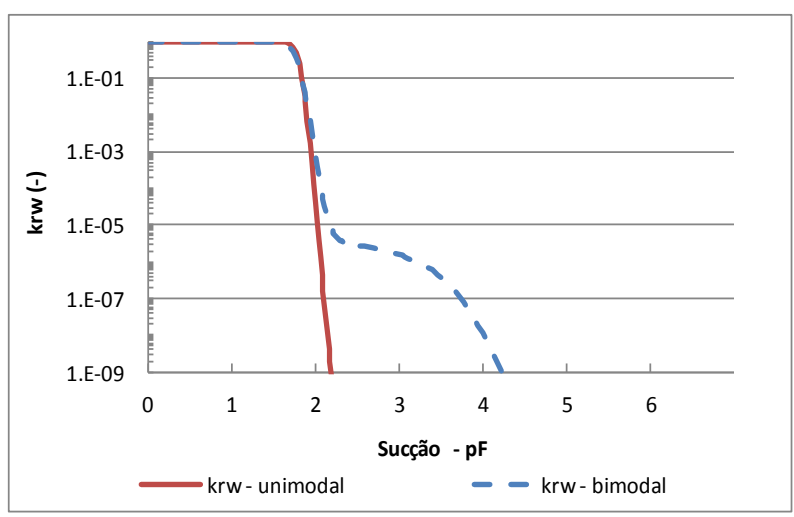

(b)

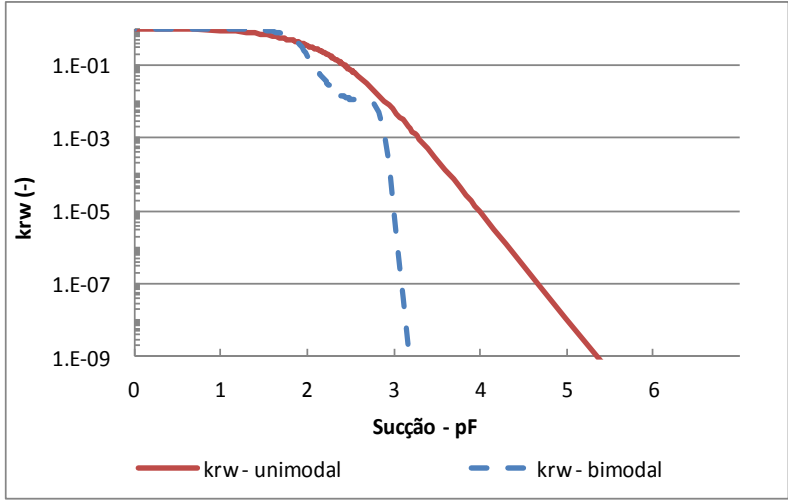

(d)

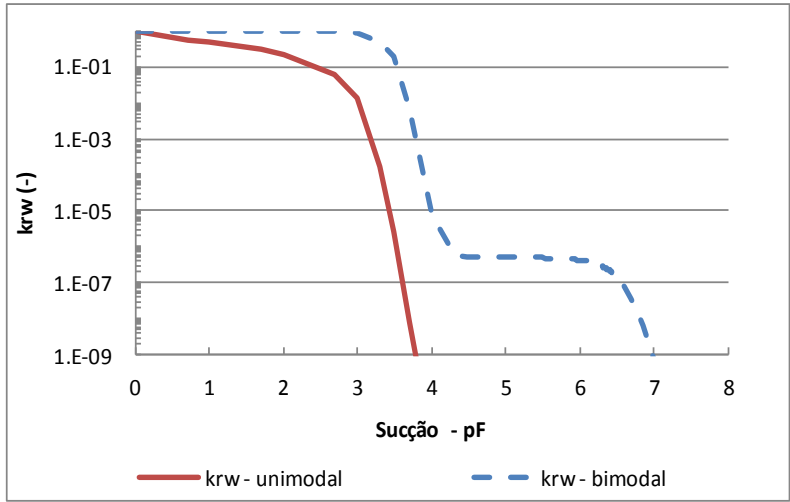

(f)

Figura 5 - Sucção versus Permeabilidade relativa para os solos das cidades de: (a) Rio Claro-SP; (b) Itirapina-SP; (c) Lins-SP; (d) Londrina-PR (MIGUEL et al., 2006); (e $e$ f) São Carlos-SP (GRACIOSA et al., 2008).

Figure 5 - Sucção versus Permeabilidade relativa para os solos das cidades de: (a) Rio Claro-SP; (b) Itirapina-SP; (c) Lins-SP; (d) Londrina-PR (MIGUEL et al., 2006); (e $e$ f) São Carlos-SP (GRACIOSA et al., 2008).

Os valores de EQM sugerem que, embora existam significativas diferenças entre a permeabilidade uni e multimodal, as discrepâncias são menores nos solos D, E e F e, diferentemente dos outros solos estudados, a queda da permeabilidade relativa é menor com o aumento da sucção, mostrando pendentes (após pressão de entrada de ar) menos íngremes com sucções superiores a $3 \mathrm{pF}$ para permeabilidades relativas superiores a $10^{-9}$.

\section{CONCLUSÕES}

Foram abordados aspectos do comportamento da permeabilidade relativa para a água como fluido molhante, a partir de métodos indiretos que se valem da curva de retenção e de modelos estatísticos de permeabilidade relativa.

Constatou-se que os solos avaliados, típicos do Brasil, apresentam distribuição bimodal de poros, e que este comportamento esteja prova- 
velmente relacionado às peculiaridades de solos mais evoluídos, como já observado por outros pesquisadores.

Devido às características bimodais desses solos, constatou-se que os métodos tradicionais podem subestimar ou superestimar a condutividade hidráulica não saturada (e consequentemente outros parâmetros que se valem desta propriedade), conduzindo a erros significativos de até duas ordens de magnitude.

Embora o presente estudo tenha sido realizado em materiais não consolidados, da zona

\section{REFERÊNCIAS}

ALFARO SOTO, M. A; CHANG, H. K; VILAR, O. M. Avaliação do escalonamento fractal de alguns solos Brasileiros. Revista Brasileira de Geociências 38, 253-263, 2008.

ASTM D6836 - 02. Standard Test Methods for Determination of the Soil Water Characteristic Curve for Desorption Using a Hanging Column, Pressure Extractor, Chilled Mirror Hygrometer, and/or Centrifuge. E2, 2008.

BACCHI, O. O., REICHARDT, K., 1993. Geometria fractal em física do solo. Scientia Agricola. Piracicaba, 50(2), 321-325. 1993.

BURDINE, N. T. Relative permeability calculations from pore-size distribution data. Petrol. Trans., AIME. 198, 71-77, 1953.

DURNER, W. Hydraulic conductivity estimation for soils with heterogeneous pore structure, Water Resour. Res., 30, 211-223, 1994.

FEUERHARMEL, C.; GEHLING, W. Y. Y.; BICA, A. V. D.; PEREIRA, A. Determinação das curvas características de solos coluvionares pelo uso combinado da placa de sucção e método do papel filtro. 5o Simpósio Brasileiro de Solos Não Saturados. São Carlos 25 a 27 de agosto de 2004. v.1, p. 243-248, 2004

GERKE, H. H., \& van GENUCHTEN, M. Th. A dualporosity model for simulating the preferential movement of water and solutes in structured porous media. Water Resour. Res. 29, 305-319, 1993.

GRACIOSA, M. C. P.; MENDIONDO, E. M.; CHAUNDHRY, F. H. Simulação hidráulica de trincheiras de águas pluviais. Revista Brasileira de Recursos Hídricos. $12(2), 89-99,2008$.

LOUGON-DUARTE, A. P.; DE CAMPOS, T. M. P.; ARARUNA, J. T. Efeitos da temperatura na relação umidade-sucção de dois solos. 5o Simpósio Brasileiro de Solos Não Saturados. São Carlos 25 a 27 de agosto de 2004. v.1, p. 235-242, 2004.

MALLANTS, D.; TSENG, P. H.; TORIDE, N.; TIMMERMAN, A.; FEYEN J. Evaluation of multimodal hydraulic functions in characterizing a heterogeneous field soil. Journal of hydrology, 95, 172-199, 1997. vadosa e com água como fluido molhante, presume-se que o mesmo comportamento pode ocorrer em aquíferos que possuam caminhos preferenciais de percolação tais como fraturas. Consequentemente, recomenda-se levar em consideração esses fenômenos quando forem utilizados métodos indiretos, que se valem da curva de retenção para a determinação da permeabilidade relativa, não somente de fluidos molhantes, mas também de fluidos não molhantes, como LNAPL, em análises de fluxo multifásico em zona saturada e não saturada.

MALLANTS, D.; JACQUES, D.; VANCLOOSTER, M; DIELS, J; FEYEN, L. A stochastic approach to simulate water flow in a macroporous soil. Geoderma, 70, 299. 314, 1996.

MARINHO, F. A. M. Medição de sucção com o método do papel de filtro. Anais... X COBRAMSEF, v. 2, p. 515 $522,1994$.

MARQUARDT, D. W. An algorithm for least-squares estimation of nonlinear parameters. Journal of the Society for Industrial and Applied Mathematics, Philadelphia, 11 (2), 431-441, 1963.

MIGUEL, M. G.; TEIXEIRA, R. S.; PADILHA, A. C. C. Curvas características de sucção de solo laterítico da região de Londrina-PR. Revista de Ciência e Tecnologia, 12 (24), 63-74, 2006.

MUALEM, Y. Hysteretical models for prediction of the hydraulic conductivity of unsaturated porous media. Water Resources Research., 12, 1248-1254, 1976.

OLIVEIRA, D. R.; GITIRANA Jr., G. F. N.; PEREIRA, J. $H$. F. Influência das propriedades hidráulicas na evaporação de solos colapsíveis não saturados. 5o Simpósio Brasileiro de Solos Não Saturados. São Carlos 25 a 27 de agosto de 2004. v.1, p. 161-167, 2004.

OTHMER, H.; DIEKKRUGER, B. and KUTILEK, M. Bimodal porosity and unsaturated hydraulic conductivity. Soil Sci., 152, 139-149, 1991.

ŠIMU゚NEK, J.; JARVIS, N. J.; VAN GENUCHTEN, M. Th. and Gärdenäs, A. Review and comparison of models for describing non-equilibrium and preferential flow and transport in the vadose zone, Journal of Hydrology, 272, 14-35, 2003

STOICESCU, J. T.; HAUG, M. D.; FREDLUND, D. G. Proceedings of the 49th Canadian Geotechnical Conference, St. Johns, Newfoundland. September 23-25. v. 2. 721-728, 1996.

SMETTEM, K. R. J.; KIRKBY, C. Measuring the hydraulic properties of a stable aggregate soil. J. Hydrology, $177,1-13,1990$

VAN GENUCHTEN, M. T. A closed-form equation for predicting the hydraulic conductivity of unsaturated soils. Soil Sci. Soc. Am. J., vol. 44, n $\square$ 5, pp. 892 - 898, 1980 